\title{
SURVEY AND MODELLING FOR THE BIM OF BASILICA OF SAN MARCO IN VENICE.
}

\author{
L. Fregonese ${ }^{\text {a }}$, L. Taffurelli a ${ }^{\text {, A. Adami }}{ }^{\text {a, }}$ S. Chiarini ${ }^{\text {a }}$, S. Cremonesi ${ }^{\text {a }}$, J. Helder ${ }^{\text {a }}$, A. Spezzoni ${ }^{\text {a }}$ \\ a Politecnico di Milano, Dept. ABC, Mantova Campus, Piazza d'Arco, 3, Mantova \\ luigi.fregonese@polimi.it, laura.taffurelli@polimi.it, andrea.adami@polimi.it, silvia.chiarini@polimi.it, \\ stefano.cremonesi@polimi.it, jacopo.helder@polimi.it, anna.spezzoni@polimi.it
}

Commission II

KEY WORDS: HBIM, modelling, Cultural Heritage, complex architecture

\begin{abstract}
:
The Basilica of San Marco is a singular case in the field of Cultural heritage, as it constitutes a construction site always active for the maintenance and preservation of the basilica itself. The continuous intervention of conservation, due to the particular environmental conditions of Venice and the opening to the public, together with the complexity of the building itself, imposes the need to identify an optimized management system. For this reason, in 2013 the Procurator of St. Marco Basilica commissioned the construction of a 3D model of the Basilica to be used for the creation of a BIM. The model must meet the required precision of the scale of 1:50, and should also include, in addition to the geometric description, a description of the mosaic and marble surfaces of the basilica through high resolution orthophoto which are essential for the restoration of the mosaics.

The complexity of the church and the large and continuous flow of public led to work in non-optimal conditions especially for the acquisition stage. The basilica has certain peculiarities that led to some important choices, for example the use of photogrammetry instead of laser scanning technique. The same technique was preferred also by the need to realize, in addition to the geometric model, high-resolution orthophotos of marbles and mosaic surfaces.

The modelling of the basilica has highlighted a number of problems related to the building features. The basilica, indeed, is the result of the juxtaposition of elements (capitals, columns) with different origin, which therefore cannot be standardized in special libraries. Moreover, especially in the extensive mosaic areas, there are not edges that characterize the architecture, but only beveled surfaces. This has resulted in a change also in the modeling paradigm with the need to identify alternative systems even for the construction of simple elements. Therefore, we decided to model using NURBS since it is the method that allows greater adherence to reality and, at the same time, a model with acceptable dimensions in terms of navigation and usability. Only the most sculptural pieces such as capitals and statues have been replaced by mesh models.

As we were interested in different elaborations, the results of this work are a geometric NURBS model for the subsequent insertion into BIM environment, with the possibility of extracting two-dimensional drawings such as plans and sections, a mesh model with low resolution textures for online navigation and high resolution orthophotos.

The survey work and modeling has been almost completed for the interior of the basilica whereas only the external remains to be acquired and modeled. For the management of the entire work it was decided to use the data organization system already used by the Politecnico di Milano for the Duomo di Milano. The BIM3DSURVEY system will be essential not only to manage the finished model, but it has been very useful also during the construction phase because it allows us to divide the entire work into more work units. However, this system required some changes to meet the peculiarity of this BIM, in particular the need to append and link the orthophotos to real surfaces, in order to use the model also as a spatial index for the images.
\end{abstract}

\section{INTRODUCTION}

\subsection{HBIM for the Basilica of San Marco}

The Basilica of San Marco has to be considered an extraordinary Cultural Heritage for many reasons. Apart from the artistic value of its architecture and pieces of art, the most outstanding characteristic of this religious complex is its liveliness. This means not only that there is a lot of visitors in each period of the year, but that the entire building and construction site is really alive. On the contrary of other churches, in San Marco there is a continuous intervention for the restoration and conservation of structures, mosaics and many details. Inside the church, for example, there are many experts who work full time as carpenter, electrician, marble worker and so on.

In this contest the knowledge of the architecture of the basilica and its state of preservation is very relevant, as it represents the guide for the interventions and the management of the whole complex. For these reasons, in 2013 the Procurator of St. Marco
Basilica decided to build the 3D model of the Basilica to be used for the creation of a BIM. This work is part of the collaborative activities between the Politecnico di Milano and the Procurator of San Marco which led, over the years, to the elevation monitoring of the basilica, the survey of the entire mosaic floor till the detailed survey and modelling of the spaces between the vaults and the roofs.

\subsection{The project}

Since the beginning, the 3D survey and modelling of the Basilica has presented many challenges connected to the project itself, the characteristics of the church and the logistic aspects The requirements of Procuratoria regarded the construction of a BIM model of the whole church, internal and external, at the scale 1:50. Moreover they demanded to use very attention to the mosaic surfaces of walls, vaults and domes (Chiabrando, 2015) . They are very interested in those surfaces as the conservationrestoration of mosaics is a very complex and frequent intervention. For the restoration of floors, for example, they 
print the orthophoto in scale 1:1 to have an exact representation of the area interested to the intervention and they can make the restoration intervention with the certainty that the final result will be the same as the initial one (Fregonese, 2006).

These requirements implied to pay attention not only to geometry for BIM modelling (Murphy, 2013), but also to colour/surface acquisition. And above all it entails to do very high resolution image acquisition for the mosaic surfaces of domes and vaults, to be able to read all single tessera even at great height.

Before starting the survey work, an important discussion concerning which type of BIM software was the most suitable for San Marco has emerged. The choice was about the use of commercial software (Revit, Archicad) (Logothetis, 2015) or a self-implemented software (De Luca, 2007). The commercial solutions guaranteed a good level of integration with other software and a good diffusion. Anyway, three years ago, they had some lacks above all in the modelling stage as they were implemented for new building, instead of existing ones. Many decorative details, capitals, vaults would have been very difficult to model in 3D way. On the opposite side, an implemented BIM system allowed to exactly customize each part of the process, but requires a lot of effort. We had also to consider the need to have a very good and accurate representation of all marble and mosaic surfaces. The element that, together with the complexity of the architecture, lead to a decision was the experience on the Main Spire of the Duomo of Milano. The 3D Survey group of Politecnico developed a BIM system - BIM3DSURVEY (Fassi, 2015) which combines the modelling issues of Rhinoceros environment, with a database structure which was particularly suitable for customization. In this BIM application, in fact, it would have been necessary to import not only the geometric model, but also images and above all orthophoto.

So, the final decision was not to use commercial BIM software, but to rely on the modelling characteristics of Rhinoceros and implement some new functions in BIM to manage (upload, view and download) images.

\subsection{The basilica and its complexity}

The basilica of San Marco has a floor plan in the shape of a Greek cross, with a dome over the crossing and another dome on each of the four arms. The nave and the transept have a central aisle and two side aisles divided by an arcade (Figure 1) The nave of the upper arm corresponds to the presbytery, raised by a few steps, below which is the crypt. To the sides of the presbytery there are two chapels.

The area of the presbytery is separated from the rest of the basilica by the iconostasis that is divided into three distinct sections: the central one, in front of the presbytery, and the two lateral parts, in front of the chapels.

The upper inner perimeter of the entire basilica, except for the apse, is crossed by the women's galleries.

A narthex wrapped around the west end disguises the cross shape but creates a wide surface for the main facade.

This architectural structure of the church and its ornaments are byzantine, but we can find also Gothic and Romanesque styles in the decorations. These are indeed the result of a continuous addition and re-use of ornaments, sculpture and precious marbles acquired during travels and conquest as a symbol of power.

One of the main distinctive feature of the basilica is just absolutely its ornamentation; composed by marble slabs, sculpture and golden mosaics, it completely hides, both inside and outside, the main brick structure of the church.
The complexity and the multitude of architectural and decorative elements that compose the basilica of San Marco involved many difficulties for the survey and modelling stage and contributed to enlarge the time of the whole work.

A certain repetition of shapes and dimensions can commonly be noted in complex architectures, but in San Marco it practically never happens. Moreover, the degradation to which it is daily subjected considering its location (high water, salinity) made almost impossible to create libraries of objects and standard elements, so each element has to be modelled as unique.

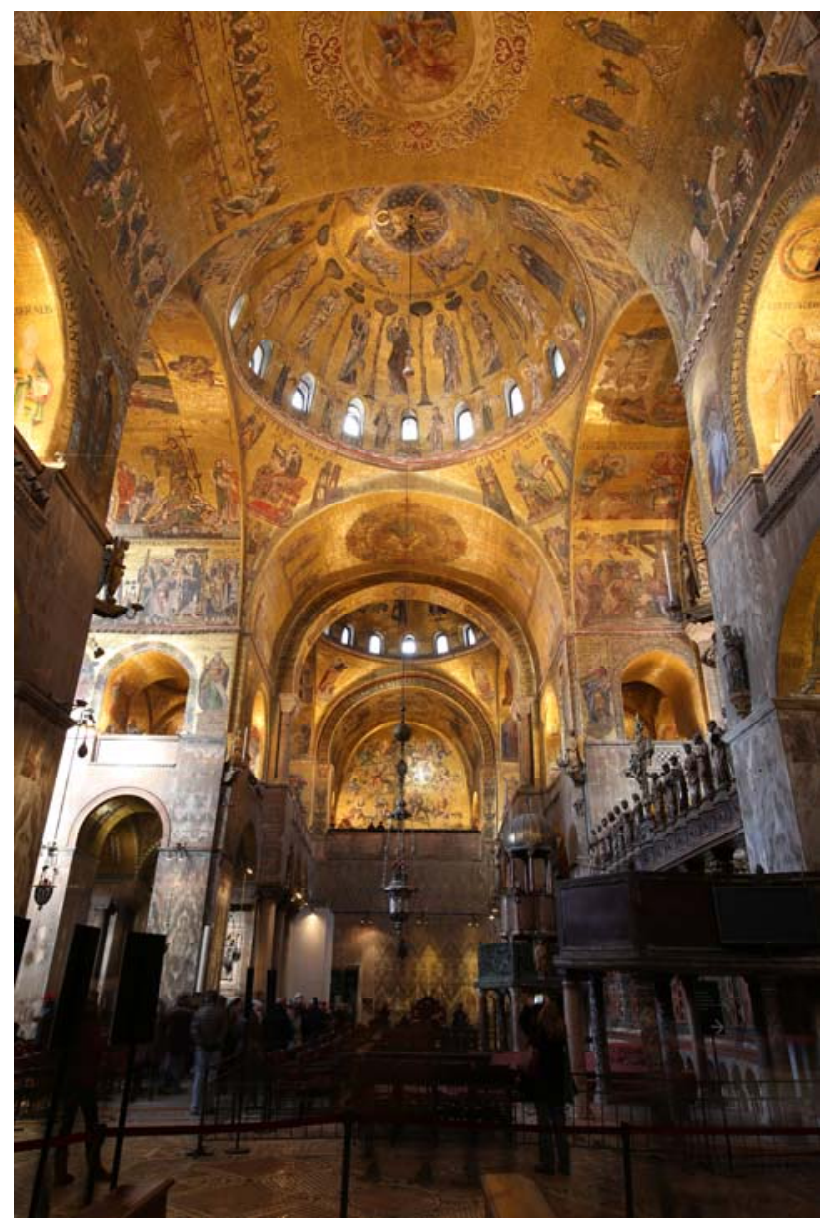

Figure 1: image of the interior of the Basilica from where we can see its complexity, the two levels of galleries, the big mosaic surfaces and the vaults and the crowd of visitors

Inside, due to the impressive gilded mosaics cover, the upper structure, composed by vaults and domes, looks like a continuous surface without edges, while the lower part of the building is completely enclosed by marble slabs and columns, in the same way as the exteriors. This is contrary to traditional architecture where the break lines are the most important element to be represented.

Moreover, the entire building presents some huge irregularity in the shape, because of a constant addiction of architectural structure and decorations during the centuries, such as the heavy wooden and lead domes overlapping the original ones or the gothic spires of the façade.

Aside from the problems generated from the architectural complexity of the basilica, there are others that are more practical and linked to the functions of the church itself and the method of survey.

The basilica is ever open to the public, some sections are just 
for praying and other for purely touristic purpose; many liturgical functions, even special ones, are done and this made the church constantly full of many people during the day.

This makes it difficult to completely close an area to work freely. It is not even allowed to work during the night because of management and economic problems.

These are the main logistical difficulties, but in parallel there are technical problems regarding instruments and materials.

Laser scanner technique generates some reactions issues with marble surfaces and gilded glass tiles. The laser signal is not correctly reflected due to the fact that is partially absorbed from the stone and moreover the mosaics surfaces causes unchecked reflections that generate errors in the point cloud obtained.

Another major problem is the light, since operating through photogrammetric survey, the need of a lighting as uniform as possible is mandatory. This action is somewhat difficult due to the co-presence of many lights, different for color, intensity and time of use: some very intense yellow lights are used in the central part of the day to allow a better visibility to touristic and to create "atmosphere". The sun which comes in through the multicolor glass windows modify the color of surfaces. All these different lights are very hard to be corrected.

More common issues, instead, are very frequent in the survey of complex architectures. Narrow spaces, to work within or to survey; scaffoldings, reducing the space even more or preventing almost completely to collect some data; weather, which may influence the measuring phase; huge volume of data, that cannot be acquired and processed without elaborating a precise and effective methodology (Fassi, 2011a).

For all these issues, generally there is not a single solution but there are different ways to operate depending on the feature of the architecture and the aim of the work.

In literature, many of these problems find a solution in an integrated approach where different kind of survey and instruments allow to adjust the reciprocal lacks (Balletti, 2015) (El Hakim, 2004). Usually geometry and color appearance are acquired in different moments and with different instruments. But in the case of San Marco, as said before, some techniques were forbidden: laser scanner.

The modelling stage put in evidence some other problems, partially yet described, which are strictly related to the complexity of the shapes. Indeed, in 3D modelling more than 2D drawing, it is very difficult to understand which is the smallest element to be represented. The rule of graphic errors, which determines that the smallest detail to be recognized in scale 1:50 has a dimension of $1 \mathrm{~cm}$, becomes a bit ambiguous in $3 \mathrm{~d}$ space.

Technologies and techniques have been refined in the last years helping to fulfil, maybe even in a faster way, the abovementioned objectives, but even so these needs can sometime become harder to reach, particularly when the target of the study is a large and complex architecture.

\section{SURVEY STAGE}

Some considerations should be done on the survey campaign preventively. All data had to be acquired during the normal San Marco opening for tourists and devotees so, comprehensibly, the survey activity required much more time than compared to work in a favourable condition. It was also necessary to plan all normal activities according with events and people flow. For this reason, all the campaign of acquisition had to be discussed with the technical office of the church and had always be interrupted for some time according to the activities of the church.

As said before, the nature of materials brought us to a specific choice in terms of survey technologies to use in this case. We decided to use only automatic multi-image photogrammetry, both for geometric and colour acquisition.

Since the beginning of the work it was evident that the survey of the basilica could not be done in a single step, but it would have been necessary to break down the entire church in small areas. So, the survey pipeline had to provide a method to do the survey step by step.

The first area we decided to acquire was the Baptistery. It represented a test bed where we tried the technologies and where we set the survey pipeline to use for the entire church. The area was optimal for test acquisition, in fact the Baptistery is a closed and limited space; it is formed from the architectural elements that are present in the whole building: domes, arches, vaults, columns, capitals, sculptures, etc. The problems faced in the test area were substantially the same of the rest of the church. Technically, in the others spaces we had to adjust the survey process and the instruments in base of the specific case, but the pipeline of survey (and modelling) was the same. For example, the dimension of the main church is higher than the Baptistery one, it requested a different lens for the acquisition phases to obtain an adapt data resolution.

Considering the necessities to acquire the geometry and the colour appearance of surfaces, we chose to acquire different series of images that allow us to obtain photogrammetric models with different details (Figure 2).
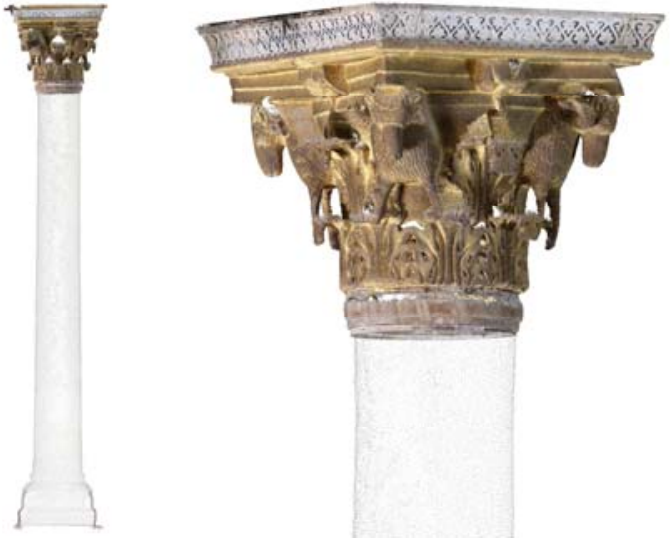

Figure 2: example of multiscale acquisition. The lower part of the column was acquired with $24 \mathrm{~mm}$ and the resulting point cloud has a less than $1 \mathrm{~cm}$ resolution. For the capital, acquired with $85 \mathrm{~mm}$, the resolution is less $1 \mathrm{~mm}$

We used three cameras (2 Canon EOS 5D mark III and 1 Canon EOS 5DS r) that allowed to works at the same times in various area and we used different lens $(24 \mathrm{~mm}-35 \mathrm{~mm}-85 \mathrm{~mm}$ $200 \mathrm{~mm}$ ). The choice of the lens was done according to the elements to acquire, the distance from the object to survey and the pixel-size we needed for the elaborations. The result was a multiscale project in which some elements are surveyed and represented at different scales. In fact, we realized generally two series of photogrammetric models: one that recreates main architectural areas using $24 \mathrm{~mm}$ or $35 \mathrm{~mm}$ lens and the other using telephoto lens for the upper mosaics surfaces and the most complex elements. Surfaces like sculptures, capitals or decorations were acquired carefully taking more images and using appropriate lens $(85 \mathrm{~mm})$ in order to obtain dense point cloud (for a mesh as complete as possible). The upper mosaic surfaces needed a very high-resolution image acquisition for the orthophotos creation considering the high distance from the 
mosaics (the domes arrive about at $28 \mathrm{~m}$ from the ground floor). All images were acquired with telephoto lens, from the tripod, from the ground level and from the women's galleries.

The church has been split in many models based on the architectural spaces (Central Nave, South Nave, Baptistery, Zen Chapel, S. Isidoro Chapel, etc.), the elements which required a detailed representation (Upper mosaics, Capital, Sculpture, etc.) and the necessity to create models with a determinate images number in the way to permit a successful data elaboration.

The different parts were geo-referenced on the basis of the existing topographic network, which was realised by the Politecnico di Milano for the basilica's floor surveys occurred in 2004-2010 (Fregonese, 2004). Using a single reference system, every new acquisition was integrated in the previous survey. The result is a database upgradable over time, georeferenced with the same general topographic network.

The general photogrammetric models were geo-referenced thanks to topographic survey by a total station (Leica TS30). In this way, they were oriented in the univocal reference system by points with known coordinates. Moreover, the topographic acquisitions allowed us to control photogrammetry model deformations, avoiding the propagation of any errors in the images alignment.

The detailed photogrammetric models were orientated using the ICP (iterative closest point) method, thus the correspondence between data have allowed to geo-reference the detailed models on the most general models, which are oriented with topography.

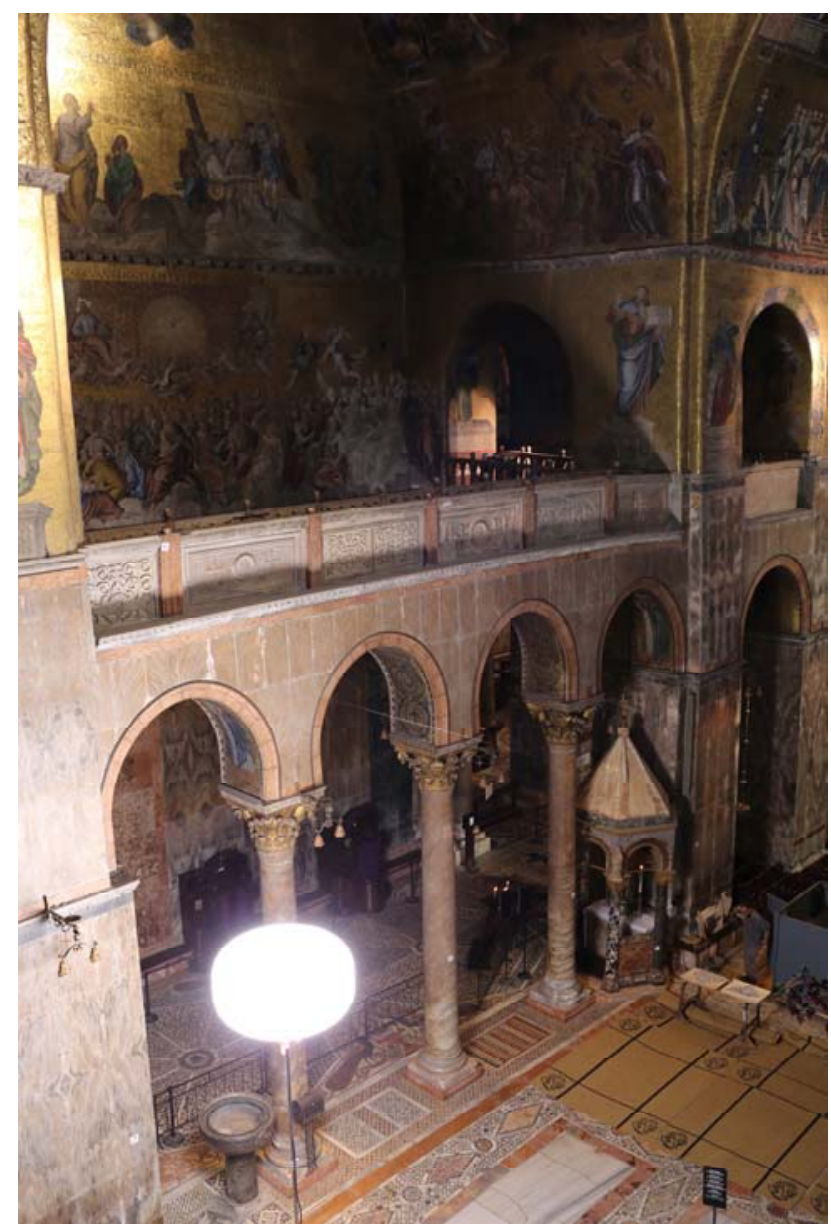

Figure 3: the lighting balloon for the photographic acquisition. It is evident that, even if the balloon is located at a certain height, the higher mosaics are not illuminated.
Automatic multi-image photogrammetry requires taking images with partial overlapping on each other and photos must have a homogeneous light to acquire realistic shots without shadows and flashes areas. To do it, we used some big lighting balloons with discharge lamp that generate neutral light, and some adjustable led spotlights more manageable than the others. Generally, the big balloons (Figure 3) were used in large spaces, where it is easier to manage them and also to illuminate high surfaces, indeed they are provided by telescopic tripods permitted us to regulate light heights. On the other hand, led spotlights are smaller and we used them in complex area where there are small spaces to work, as for the galleries level, and where it was necessary to correct the illumination coming from different directions to obtain a homogeneous condition.

Mainly, the major difficulty was to obtain a diffuse illumination in large spaces, trying to contrast the natural light coming from the windows, the church's artificial illumination and the related shadows.

After the acquisition phases, the images were processed with the photogrammetric software Photoscan by Agisoft in the wellknown pipeline. The dense point clouds of the surveyed areas, are the main results of the acquisition (Figure 4).

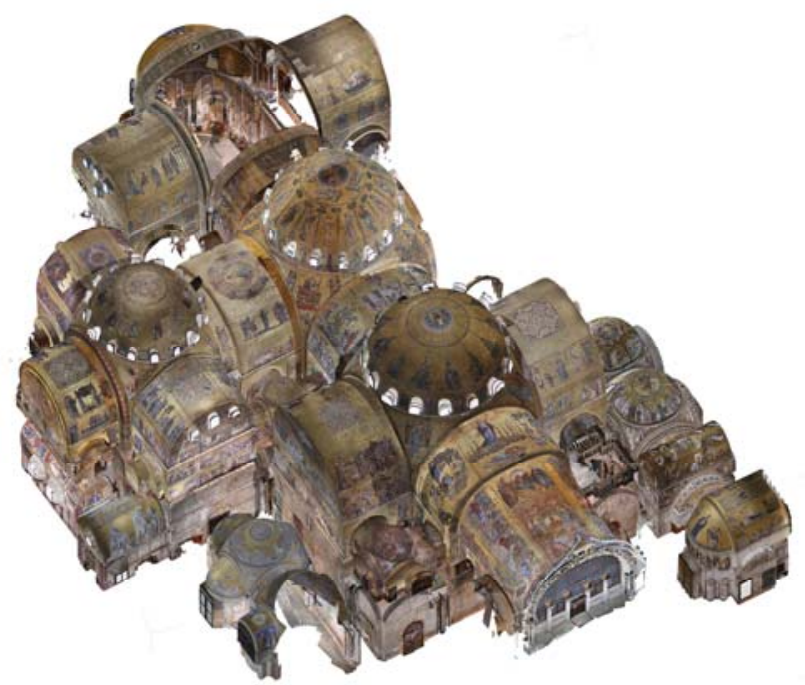

Figure 4: Merging of all calculated point clouds of the interior of the basilica

\section{MODELLING STAGE}

To choose the ideal type of modelling process it was necessary to understand which kind of 3D object (like surfaces, solids, NURBS) was the ultimate way to describe each type of architectural component of the building. Therefore, it was necessary to analyse the different architectural features of the basilica and its detail.

Because of the complex shape of the interior, specially referred to the continuous surface of the vaults, it was difficult to individuate architectural or structural elements which can be described with solid object, as a matter of fact, all we can see is a complex, thick and stratified decorated surface. Furthermore, the whole decoration was composed by a huge variety of pieces, all different between them because they are the result of a continuous addition and re-use of decorations, sculpture and precious marbles acquired during travels and conquest, like a sort of monumental collection. This particular aspect makes almost impossible to create standard objects or libraries that can be employ to simplify the modelling process. Each object 
should be treat like a unique one, even if this approach is quite unusual in BIM applications.

We considered surface modelling as the most flexible and effective method to describe the complexity of the basilica. Moreover, this choice permitted to complete and make available blocks of the model without the necessary of having the whole survey of the adjacent area of the building. This peculiarity allows to test the realization process from the survey to the texturing of the modelled object, individuating the guide-lines for the successive areas, like in the case of the baptistery.

NURBS model seemed to be the best solution to obtain a great adherence to reality, especially considering the complex and irregular surface of the mosaics and the deformations and not perpendicular shape of the walls and structures. Moreover, this kind of modelling process allows to obtain a complex and accurate model, with an adequate dimension in terms of navigation and usability, which means objects with the smallest possible size without the loss of details precision considering the representation scale of the model.

The amount of data, necessary to describe a large building like the basilica of San Marco, made difficult to use and navigate the $3 \mathrm{D}$ model without a subdivision in smallest parts of some areas. To allow the management of the complete model, and to control and make a correct junction of the areas and the architectural component of it, it was necessary to share the work between all components of the working group. For this purpose, we decided to apply the feature of Rhinoceros (the software selected for modelling) called "worksession". This is basically a base file which manages a list of 3D models and allows multiple users to navigate and work on them like it is a unique one, avoiding that the dimension (byte) of the original files makes the process more demanding.

As we saw before, the variety of elements did not permit to create "standard" objects, but they could be grouped by similar features and, for each group, a standard methodology of modelling has been individuated. Each method is based on the fundamental concept of the extraction of generating section of the object from the photogrammetric point cloud. In practical terms, the point clouds extracted from the photogrammetric models were converted in Pointools file format (.pod) and then imported in Rhinoceros. The Pointools plugin allows to extract new views and sliced from the point-clouds directly in Rhinoceros environment, to build the generating curves.

For the main structure of the walls, the aim was to obtain a simple surface which could describe the complex shape of the object, by the extraction of some control curves in strategic points, like the junction of the marble slabs or the connection with cornices, columns and other decorations. The same approach was used for the construction of small objects made by simple surfaces or objects with a constant geometric profiles (Figure 5). In these category we included the marble cornices, even if they have a sculptural fine decoration. This choice was due both to avoid a large use of meshes and to respect the representation scale chosen for the 3D model (1:50) At this scale, in fact, some tiny decorations, smallest than 1 centimetre, could be omitted in the model. This information, mainly connected to the aspect of the object anyway is given by the textures and orthophotos.

So, for each object was extracted a geometric construction section, which was modified in terms of dimension, rotation and distortion to fit the point cloud. The modifications were applied with a frequency based on the extension of the object, its deformations and the presence of key points like corners, fractures and junctions.

A complete different approach has been employed for the sculptural objects like capitals and statues. In these cases, we went a little further the scale of representation imposed for the rest of the model, both because these kinds of object need a particular attention and for the extreme complexity of their shape, that did not allow a geometric simplification. In these cases, the NURBS model gave way to a mesh model directly extract from the dense point cloud and triangulated and processed in Rapidform.

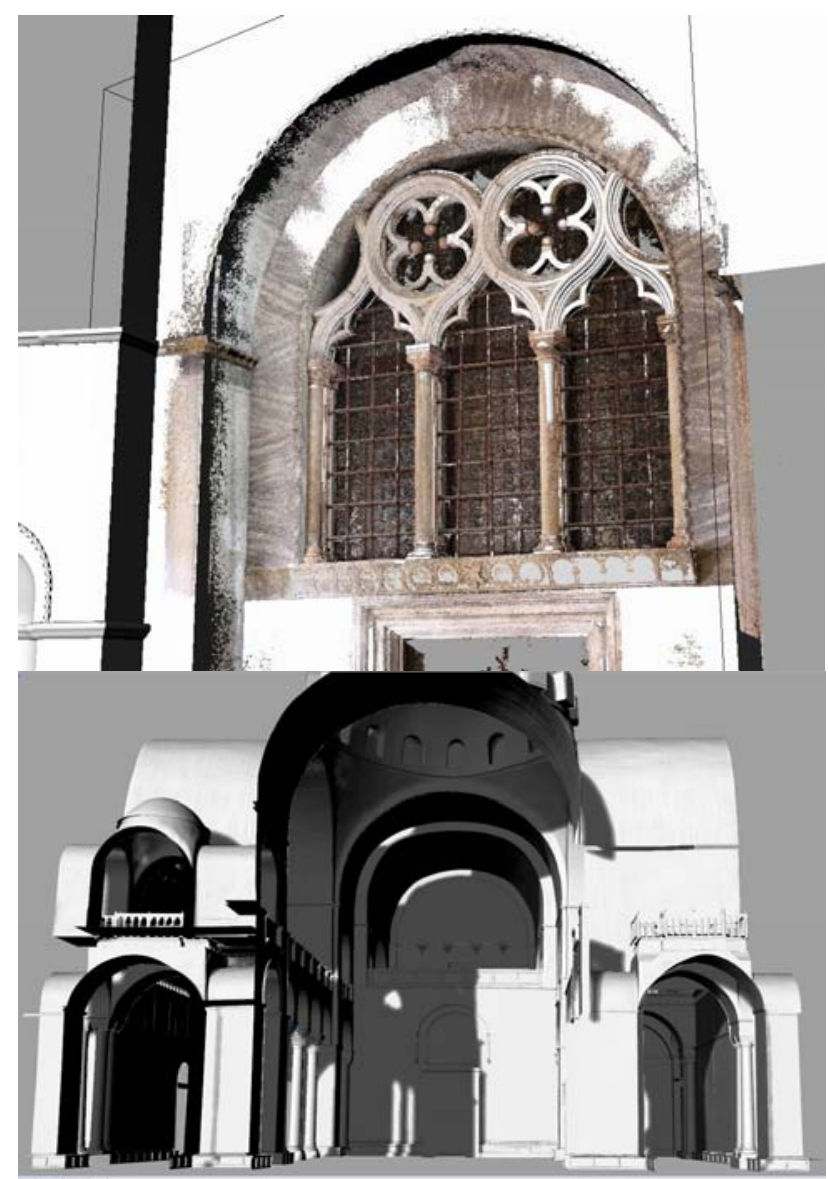

Figure 5: (top) the gothic window of Cappella Zen, is modelled by using sections extracted from the corresponding point cloud. In the image point cloud and NURBS model are overlapped. (bottom) general view of the model

The complex surface of the vaults was obtained by an interpolation of section extracted from the data using a reference grid (Figure 6). The outline and the pace of the grid depended on the dimension, the geometrical features and the complexity of the shape that had to be obtained. For example, a simple and quite smooth barrel vault requires a regular square grid with $50 \mathrm{~cm}$ step, instead a small dome in a pillar (each pillar has a structure similar to the nave, with a little dome and four smaller pillars) with its pendentive, required a radial pattern grid in one direction, and a close series of horizontal section that can reach a 20 centimetre or less distance.

As the extraction of the model was finished, the 3D model obtained needed to be divided in singular elements, which should be classified with a code and assigned to a layer to be used in BIM3DSURVEY system. The classification was made according to the typology of architectural component, for example: vault, arch, dome, wall, cornice, column, floor and windows.

Once each object has been modelled and classified, it needed to be completed with the application of RGB data. To achieve this, 
the NURBS object, in our case built in Rhinoceros, was converted into a polygonal model and was used in the photogrammetric software Agisoft.

The mesh object obtained was imported in the original photogrammetric model and used. The texture of the object was obtained by projecting the original oriented images used for the construction of the dense point cloud on the object surface. This step, as all the other stage of modelling, is very far to be automated and required an intervention to select only the best images to be used.

Not all the cameras were necessary to realize the orthophoto. We made a first selection by checking the quality of the images, to keep the best ones and to control the pixel size prefixed. Once the best quality images were selected, we decided to use only the most nadir cameras, to avoid an excessive stretching of pixels. Finally, the pictures with inhomogeneous light conditions were discarded to achieve the best possible radiometric information which affect the general quality of the orthophoto.

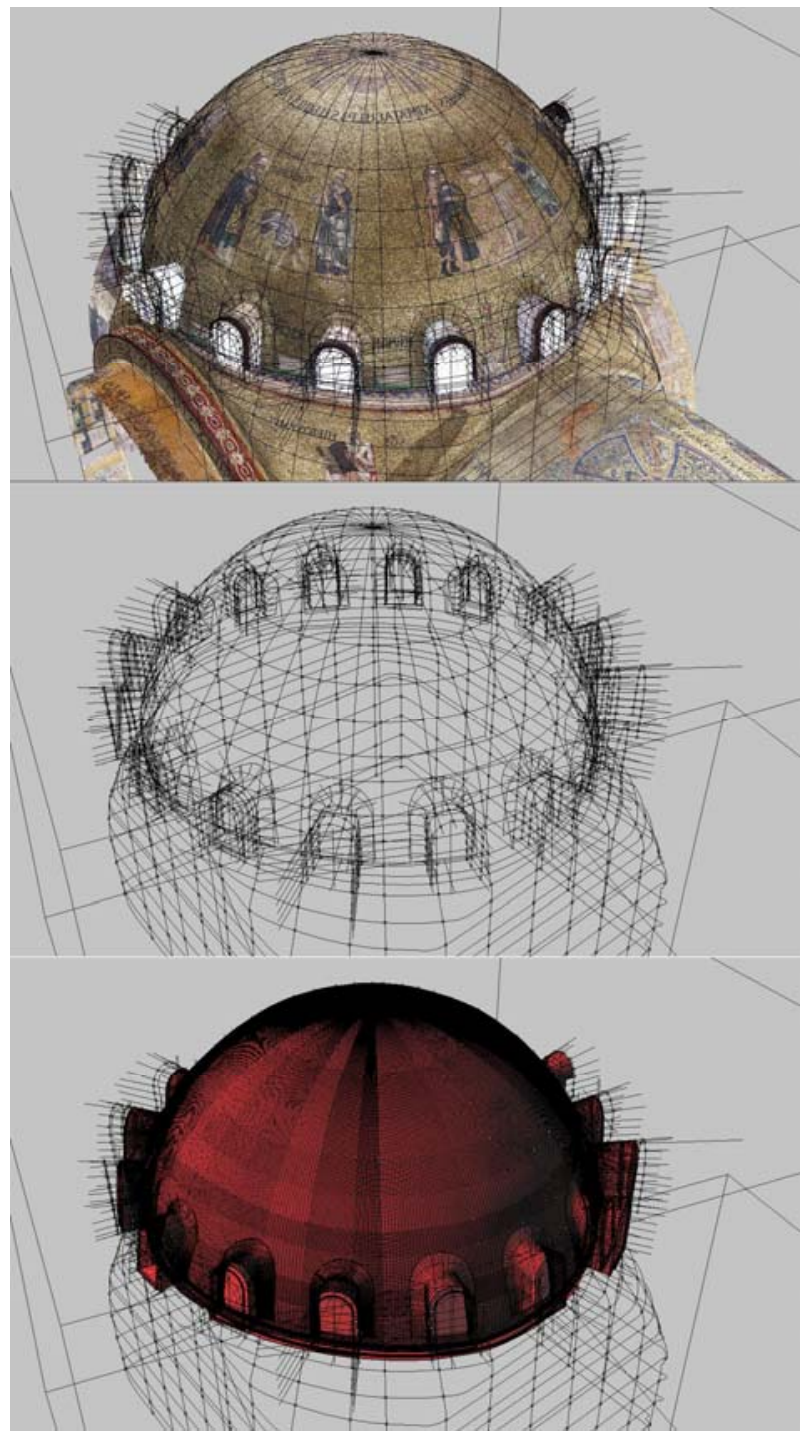

Figure 6: for the most complex vaults and domes, a network of sections was extracted from the point cloud and is used to build the geometric model

The orthophoto was produces with a pixel size of 0.0005 meters which allowed to see clearly the border of the tiniest mosaic tiles, so it can be used for restoration or analysis purposes
(Figure 7).
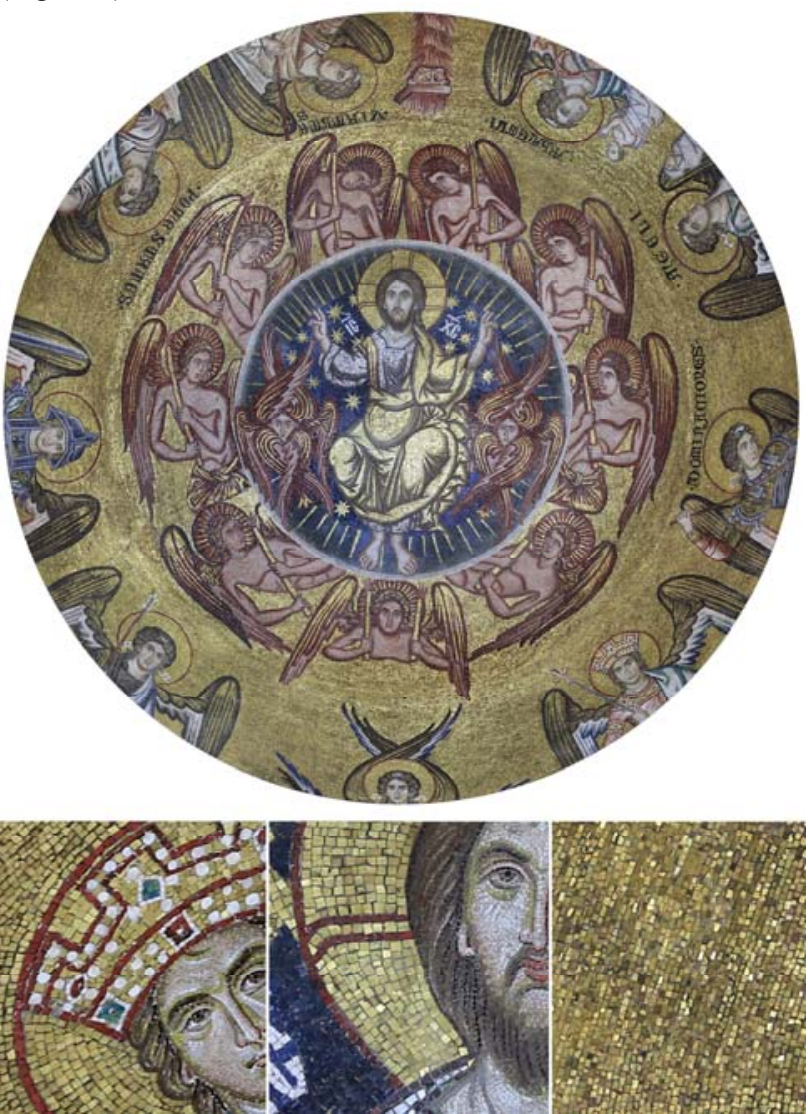

Figure 7: orthophoto of the dome of Baptistery with a detail where we can see each tessera. The resolution is $0.0005 \mathrm{~m}$ for pixel

\section{RESULTS FOR BIM APPLICATION}

At the end of the process, the results of the whole process are three kind of products (Figure 8):

a) a geometric NURBS model for the subsequent insertion into BIM environment, with the possibility of extracting twodimensional drawings such as plans and sections;

b) A mesh model with low resolution textures for online navigation;

c) A high resolution orthophoto.

These three results represent the answer to the requirement of the customer. The NURBS model will be used especially in the BIM application to link the database (about history, restoration, planned conservation). It guarantees a good accuracy of the model, but, at the same time, it is not so difficult to manage in terms of memory occupation and file size. In Rhinoceros it is possible, as in other BIM software, to extract plans and elevations, directly from the $3 \mathrm{D}$ model. This is a very relevant function for the management offices of the Basilica, as it allows to have innumerable sections and plans of each part and element of the architecture.

The mesh model can be considered as a by-product of the pipeline. To obtain the orthophoto it was necessary to use a mesh model in Agisoft. So we converted the 3D NURBS model into a mesh model and we decided to texture it, even if with low resolution texture, in order to furnish also a tool which is most 
suitable for a virtual navigation.

Finally, the orthophoto represents the other request of the Procuratoria of San Marco. The high resolution allows to recognise each single tessera of the mosaic, but they are not so easy to manage because of their file size.

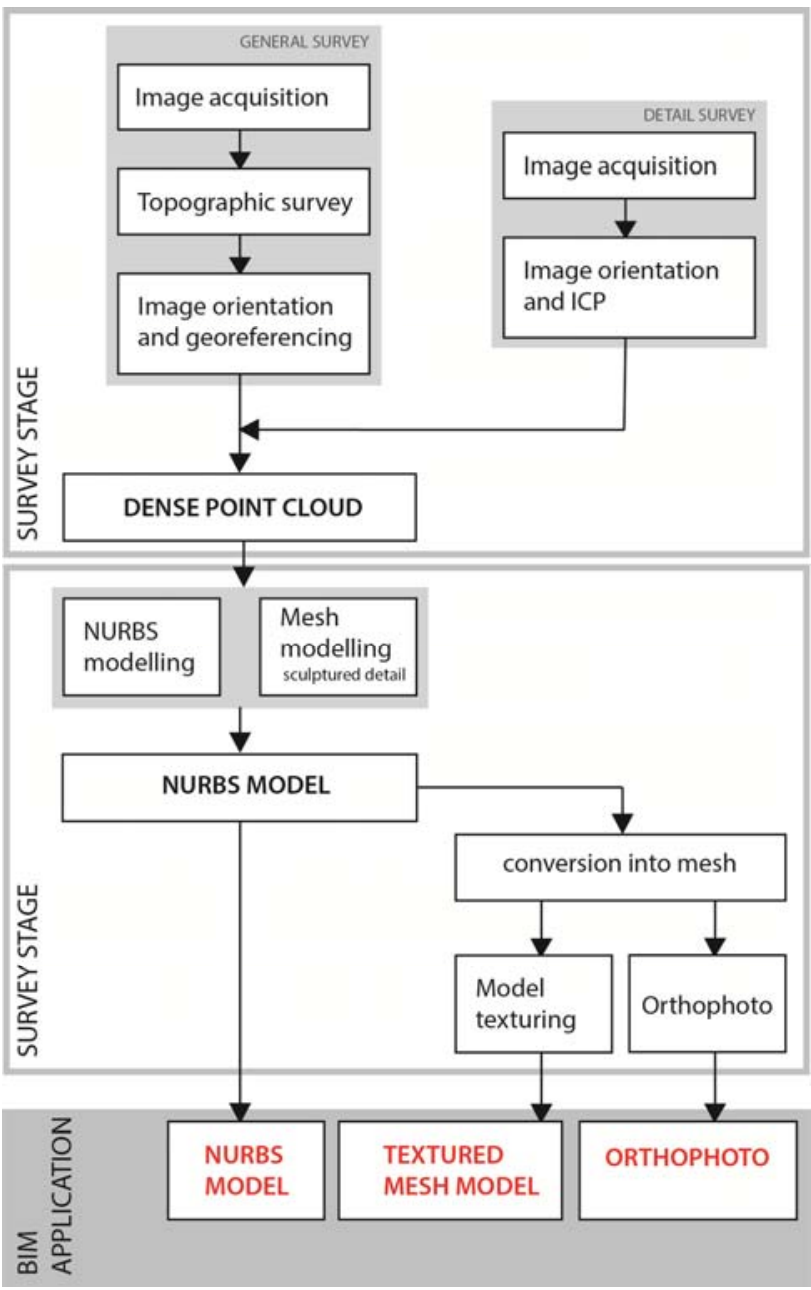

Figure 8:workflow of acquisition and modelling stage

To handle these three kind of results (Figure 9) strictly connected but independent from each other, the BIM system, BIM3DSURVEY, developed by Politecnico di Milano for the main spire of the Duomo of Milano, was selected. It allowed to manage easily big dimension 3D models, so it was particularly suitable for the architecture of San Marco. It maintains a strict relationship with the original Rhinoceros model so it is possible to update the final model or add new part, not involved in this work. Furthermore, it allows to link the uploaded model to many other information. For this application, the system has been implemented with a function which allow to use the model as a 3D spatial index for the orthophoto. The user can select an element and then it is possible to view and download the orthophoto of that element.

\section{CONCLUSIONS}

This research concerns the survey and modelling work of the Basilica of San Marco in Venice to build a BIM of the architectural complex. This paper, placed in time almost to the conclusion of the work on the interior of the basilica, describes the difficulties in the construction of the 3D model for a BIM application. In particular, it focuses on many problems, both logistic and technical, connected with the unique characteristics of the Basilica of San Marco. It is evident that in a such complex architecture, the technical problems are only a part, and that also logistic difficulties play an important role. It happens, indeed, that the problems of accessibility, of movement in space and their management, of coexistence of different activities, determine new strategies for data acquisition and don't allow to apply the most widespread methodologies or pipelines. To solve this problem, the existing topographic network was very important, as it allowed to work on different part of the church, not always neighbouring. Also in terms of global accuracy, the use of the existing topographic network (previously materialized and adjusted) permitted to have a general control of errors and not to concentrate them in a single area.
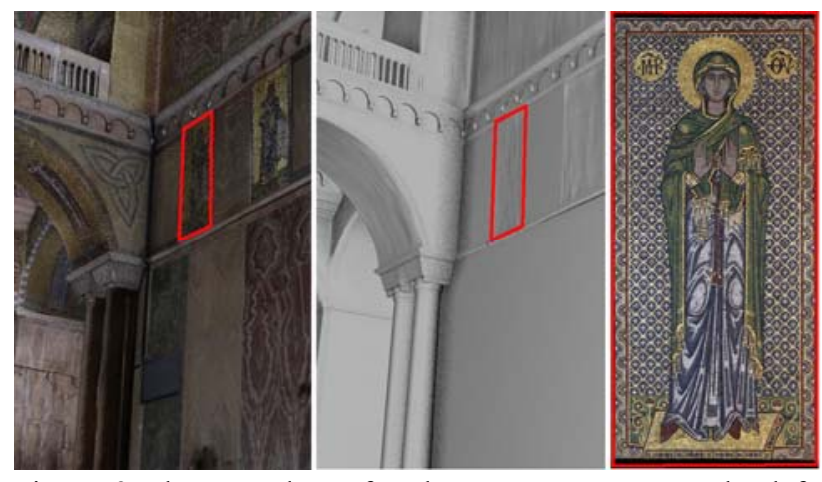

Figure 9: three products for the same area. From the left: textured mesh, NURBS model, Orthophoto

With reference to survey techniques, photogrammetry confirmed its usability and suitability also in complex contexts. In the case of San Marco, where laser scanner was not suitable, photogrammetry was used not only to acquire the colour and the appearance of surfaces, but also to obtain their geometric shapes. It is mandatory, in these application, to work with a predefined pipeline, in order to maintain, as much as possible, the same geometry of acquisition, the same distances and parameters. The use of the topographic network helps to take advantages of the photogrammetric process because we can build single local photogrammetric models, but correctly georeferenced in a single reference system.

Some problems cannot be completely solved. The survey lighting conditions are not the ideal ones. The solution of using big lighting balloons to have a global and diffuse light on the surfaces, according to the use of smaller led light projectors, allowed to have a sufficiently homogeneous light.

As concerning the modelling stage, the NURBS approach confirmed its suitability for this kind of complex architecture. Also in this context, the big problem is the fact that the modelling work cannot be done in a single step on the basilica, but it has to be split in many parts and moments. It implied that the model had to be elaborated in different times: the first elaboration is the construction of the model. And then it was necessary to adapt the model of the part to the neighbouring parts. Another difficult aspect is the dimension of the file. It was difficult, even using workstations, to maintain all modelled elements in a single file, so it was very useful to work, in Rhinoceros, with the "work session" option: it allows to connect different files in a single environment.

In terms of geometric modelling, it was very difficult to define the level of detail for the 3D model. The theory helps in the 
definition of the smallest visible detail for each scale of representation but it doesn't reach a unique rule. The only way to solve this problem, was to try on a testbed different resolution in order to find the most suitable one for the required scale.

The final consideration regards the management of the results of the survey. In this way, according to the requirement of the contractor and the working pipeline, we obtained three elaborations for each element of the basilica: a geometric NURBS, a textured mesh model and high resolution orthophotos. We chose to manage these products in a complex system which is the BIM3DSURVEY, developed by the Politecnico di Milano for the main spire of the Duomo of Milano, and modified for the Basilica di San Marco, especially for the management of the orthophotos.

\section{REFERENCES}

Balletti, C., Guerra, F., 2015: The survey of cultural heritage: a long story Rend. Fis. Acc. Lincei 26 (Suppl 1): 115.

Chiabrando F., Donadio E., Rinaudo F., 2015: SFM for orthophoto generation: a winning approach for cultural heritage knowledge in The International Archives of the Photogrammetry, Remote Sensing and Spatial Information Sciences, Volume XL-5/W7, 2015 25th International CIPA Symposium 2015, pp. 91-98.

De Luca L., Bussarayat C., Stefani C., Véron P. and Florenzano M., 2007: An Integrated Framework to Describe, Analyze, Document and Share Digital Representations of Architectural Buildings in Proc. 8th International Symposium on Virtual Reality, Archaeology and Cultural Heritage (VAST), pp. 2427, D. Arnold, F. Niccolucci, A. Chalmers (Eds), UK.

El Hakim S., Beraldin J.A., Picard M., and Godin G., 2004: Detailed 3D Reconstruction of Large-Scale Heritage Sites with Integrated Techniques in IEEE Computer Graphics and Applications ,Volume: 24, Issue: 3, May-June 2004

Fassi F., Achille C., Gaudio F., Fregonese, L., 2011: Integrated strategies for the modelling of very large, complex architecture in The International Archives of the Photogrammetry, Remote Sensing and Spatial Information Sciences, Vol. XXXVIII-5, pp $105-112$

Fassi F., Achille C., Fregonese L., 2011: Surveying and modelling the Main Spire of Milan Cathedral using multiple data sources in The Photogrammetric Record 26(136):462 487 - December 2011

Fassi F., Achille C., Mandelli A., Rechichi F., Parri S., 2015: A new idea of BIM system for visualization, sharing and using huge complex 3D models for facility management. The International Archives of the Photogrammetry, Remote Sensing and Spatial Information Sciences, Avila, Spain, Volume XL5/W4, pp. 359-366.

Fregonese L., Taffurelli L., 2004: Il pavimento musivo della Basilica di San Marco a Venezia: ortofoto digitale 3D a grande scale a supporto dell'attività di tutela, di progetto e di cantiere. E-Arcom 2004 - Tecnologie per comunicare l'architettura. Ancona, Italy.

Fregonese L., Monti C., Monti G., Taffurelli L., 2006: The St. Mark's Basilica pavement. The digital ortophoto $3 \mathrm{~d}$ realisation to the real scale $1: 1$ for the modelling and the conservative restoration in Innovations in 3D Geo Information Systems, First International Workshop on 3D Geoinformation, 7-8 August, 2006, Kuala Lumpur, Malaysia

Logothetis S., Delinasiou A., Stylianidis E., 2015: Building Information Modelling for cultural heritage: a review. ISPRS Annals of the ISPRS, Volume II-5/W3, 2015 25th International CIPA Symposium 2015, 31 August - 04 September 2015, Taipei, Taiwan, pp. 177-183

M Murphy M., McGovern E., Pavia S., 2013: Historic Building Information modelling - adding intelligence to laser and image based surveys. The International Archives of the Photogrammetry, Remote Sensing and Spatial Information Sciences, Volume XXXVIII-5/W16, 2011 ISPRS Trento 2011 Workshop, 2-4 March 2011, Trento, Italy.

Remondino, F., Spera, M. G., Nocerino, E., Menna, F., Nex. F., 2014.: State of the art in high density image matching. The Photogrammetric Record, 29.146, pp. 144-166

Saygi, G., Remondino, F., 2013: Management of Architectural Heritage Information in BIM and GIS: State-of-the-art and Future Perspectives. International Journal of Heritage in the Digital Era, Vol.2(4), pag. 695-714.

Simeone, D., Cursi, S., Toldo, I. \& Carrara, G., 2014: B(H)IM Built Heritage Information Modelling. Extending BIM to historical and archeological heritage representation. Fusion, Proceedings of the $32^{\text {nd }}$ International Conference on Education and research in Computer Aided Architectural Design in Europe, 613-622. Vol. 1. eCAADe: Conferences 1. Newcastle upon Tyne, UK: Northumbria University, 2014.

Volk R., Stengel J., Schultmann F., 2014: Building Information Modelling (BIM) for existing buildings - Literature review and future needs. Automation in Construction, 38, 109-127. 